\title{
Minimal Sets with Maximum Cardinality
}

\author{
D.K.Thakkar ${ }^{1}$ \\ Department of Mathematics \\ Saurashtra University, Rajkot \\ dkthakkarl@yahoo.co.in
}

\author{
K.N.Kalariya ${ }^{2}$ \\ Department of Mathematics \\ V.V.P. Engineering College, Rajkot \\ kiritkalariya@gmail.com
}

\begin{abstract}
In this paper we consider minimal H-dominating sets with maximum cardinality which is called upper H-dominating sets in a hypergraph. We prove several theorems regarding the effect of removing a vertex from the hypergraphs on the upper H-domination number of the hypergraph. For this purpose we consider a sub-hypergraph and partial sub-hypergraph of the given hypergraph.
\end{abstract}

Keywords: H-dominating set, H-domination number, Minimal H-dominating set, Upper H-dominating set, Upper H-domination number.

AMS Subject Classifications: 05C99, 05C69, 05 C07.

\section{INTRODUCTION}

The concept of a hypergraph is an extension of a graph. It may be interesting to define the concepts for the hypergraphs which have already been defined for graphs. In addition to this it may be possible to define concepts for hypergraphs which have property that the they are the familiar concepts for graphs. For example in [3], we have defined H-dominating sets for hypergraphs. This concept coincides with the concept of domination for graphs. This concept is stronger than domination for hypergraphs and in particular the H-domination number of a hypergraph is at least as big as the domination number of the hypergraph. In this paper we consider a minimal $\mathrm{H}$-dominating sets with maximum cardinality. These sets are called upper H-dominating sets in the given hypergraph. The cardinality of such a set is called the upper H-domination number of the hypergraph.

Let $G$ be a hypergraph and $v \in V(G)$ such that $\{v\}$ is not an edge of $G$. Consider the set $V(G) \backslash\{v\}$. This set induces two types of hypergraph G: (1) The sub-hypergraph $G \backslash v$ and (2) The partial subhypergraph $G \backslash v$. Our theorems in this paper are about upper H-dominating sets in the hypergraph $G$ and in the hypergraphs $G \backslash v$ mentioned above. In particular we prove necessary and/or sufficient conditions under which the upper $\mathrm{H}$-domination number decreases or remains same. Before this we prove that the upper $\mathrm{H}$-domination does not decrease when a vertex $v$ is removed from the hypergraph.

\subsection{Definition and Notations:}

Definition 1.1.1 (Hypergraph)[2]: A hypergraph $G$ is an order pair $(V(G), E(G))$ where $V(G)$ is a non- finite set and $E(G)$ is a collection of nonempty subsets of $V(G)$.

Definition 1.1.2 (Dominating set, Minimum dominating set, Domination number) [1]: Let $G$ is a hypergraph. A set $S \subseteq V(G)$ is said to be a dominating set if for every vertex $v$ in $V(G) \backslash S$, there is a vertex $u$ in $\mathrm{S}$ such that $v$ is adjacent to $u$.

Let $G$ be a hypergraph. A dominating set with minimum cardinality is called minimum dominating set or a $\gamma$-set. The cardinality of a minimum dominating set is called the domination number of the hypergraph and it is denoted as $\gamma(\mathrm{G})$.

Definition 1.1.3 (H-dominating set) [3]: Let $G$ is a hypergraph. A set $S \subseteq V(G)$ is called $\mathrm{H}$ dominating set if for each vertex $v$ in $V(G) \backslash S$, there is an edge $e$ containing $v$ such that $e \backslash\{v\}$ is subset of $\mathrm{S}$. 
Definition 1.1.4 (Minimum H-dominating set, H-domination number)[3]: An $\mathrm{H}$ - dominating set $\mathrm{S}$ of a hypergraph $G$ is said to be a minimum $\mathrm{H}$-dominating set if its cardinality is minimum among all H-dominating sets of $G$.

The cardinality of a minimum H-dominating set is called the H-domination number of the hypergraph $G$ and its denoted by $\gamma_{\mathrm{h}}$. A minimum H-dominating set is also called a $\gamma_{\mathrm{h}}$-set of $G$.

Definition 1.1.5 (Upper H-dominating set, Upper H-domination number): A minimal $\mathrm{H}$ dominating set with maximum cardinality is called a $\Gamma_{h}$-Set. The cardinality of a $\Gamma_{h}$-set is called the upper H-domination number of $G$. And it is denoted as $\Gamma_{h}(G)$.

Definition 1.1.6 (Sub-hypergraph) [2]: Let $G$ is a hypergraph and $\mathrm{H}$ is a nonempty subset of $V(G)$. The sub-hypergraph induced by the vertices of $S$ is the hypergraph whose vertex set is $S$ and the edge set. $E(S)=\left\{e^{\prime} \neq \emptyset: e^{\prime}=e \cap S\right.$, for some edge e of $\left.G\right\}$.

Definition 1.1.7(Partial sub-hypergraph)[2]: The partial sub-hypergraph induced by the vertices of $\mathrm{S}$ is the hypergraph whose vertex set is $\mathrm{S}$ and edge set $E(S)=\{e \in E(G) / e \subseteq S\}$.

We make some conventions about the hypergraph considered in this paper.

(1) The empty set will not be regarded as an edge of a hypergraph.

(2) In every hypergraph $G$ the number of vertices in any edge is at least 3 and the number of vertices in any edge of the sub-hypergraph $G \backslash v$ is at least 2 .

(3) If $e$ and $f$ are edges of $\mathrm{G}$ and $e$ is a subset of $f$ then $e$ must be a singleton set.

(4) If $x$ and $y$ are distinct vertices of $\mathrm{G}$ then there is at most one edge $e$ which contains both $x$ and $y$.

\section{Minimal Sets With Maximum Car dinality}

\subsection{Sub-hypergraph $G \backslash v$}

With H-domination in hypergraphs there are associated two numbers namely $\mathrm{H}$-domination number $\left(\gamma_{h}\right)$ and upper H-domination number $\left(\Gamma_{h}\right)$. Now we prove some results about upper H-domination number of hypergraph

Let $\mathrm{G}$ be a hypergraph and $v \in V(G)$ consider the sub hypergraph $G \backslash v$. We have the following Proposition.

Proposition 2.1.1: If $\mathrm{S}$ is a minimal $\mathrm{H}$-dominating set of $G \backslash v$ which is not an $\mathrm{H}$-dominating set of $\mathrm{G}$ then $S \cup\{v\}$ is a minimal H-dominating set of $\mathrm{G}$.

Proof: First we prove that $S \cup\{v\}$ is an H-dominating set of G. Let $w$ be a vertex which is not in $S \cup\{v\}$ then $w$ is a vertex in $G \backslash v$ which is not in $\mathrm{S}$. Let $e^{\prime}$ is an edge of $G \backslash v$ such that $e^{\prime} \backslash\{w\}$ is a subset of $\mathrm{S}$. Let $e$ be an edge of $\mathrm{G}$ such that $e \backslash\{v\}=e^{\prime}$ then $e \backslash\{w\}$ is a subset of $S \cup\{v\}$. Thus $S \cup\{v\}$ is an H-dominating set of $G \backslash v$. Let $S_{1}=S \cup\{v\}$. Since $\mathrm{S}$ is not an H-dominating set of $\mathrm{G}$ there is no edge $e$ such that $e \backslash\{v\}$ is a subset S. therefore there is no edge $e$ containing $v$ such that $e$ is a subset of $\mathrm{S}_{1}$. Therefore $v$ belongs to private neighborhood of $v$ with respect to $\mathrm{S}_{1}$. Let $u \in S_{1}$ such that $u \neq v$ then $u \in S$. suppose there is an edge $f$ in G containing $u$ such that $f$ is a subset of $S_{1}$. Let $f^{\prime}=f \backslash\{v\}$ then $f^{\prime}$ is an edge of $G \backslash\{v\}$ containing $u$ such that $f^{\prime}$ is a subset of S. Thus we have proved that if there is no edge in $G \backslash v$ containing $u$ which is a subset of $\mathrm{S}$ then there is no edge in $\mathrm{G}$ containing $u$ which is a subset of $S_{1}$. Therefore if $u$ belongs to private neighborhood of $u$ with respect to $\mathrm{S}$ then $u$ belongs to private neighborhood of $\mathrm{S}_{1}$ in $\mathrm{G}$. Suppose $u \in S$ and $u \neq v$. Suppose there is a vertex $w$ in $G \backslash v$ such that $w \neq u$ and $w$ belongs to private neighborhood of $\mathrm{u}$ with respect to $\mathrm{S}$.

(1) There is an edge $f^{\prime}$ in $G \backslash v$ containing $w$ such that $f^{\prime} \backslash\{u, w\}$ is a subset of $\mathrm{S}$ then $f \backslash\{u, w\}$ is a subset of $\mathrm{S}_{1}$.

(2) Suppose $h$ is an edge of G containing $w$ such that $h \neq f$ then $h^{\prime} \neq f^{\prime}$ where $h^{\prime}=h \backslash\{v\}$. Now $h^{\prime}$ contains a vertex of $G \backslash v$ which is not in $\mathrm{S}$ therefore $h$ also contains a vertex of $\mathrm{G}$ which is not in $\mathrm{S}_{1}$. Thus $w$ belongs to private neighborhood of $u$ with respect to $S_{1}$ in G. 
Thus we have proved that for every vertex $x$ in $\mathrm{S}_{1}$ private neighborhood of $x$ in $\mathrm{S}_{1}$ is a nonempty subset of $\mathrm{G}$. Thus $\mathrm{S}_{1}$ is a minimal H-dominating set of $\mathrm{G}$. Hence the theorem is proved.

Proposition 2.1.2: Let $\mathrm{G}$ be a hypergraph and $v \in V(G)$. If $\mathrm{S}$ is a minimal $\mathrm{H}$-dominating set in the sub-hypergraph $G \backslash v$ and $\mathrm{S}$ is also an $\mathrm{H}$-dominating set of $\mathrm{G}$ then $\mathrm{S}$ is a minimal $\mathrm{H}$-dominating set of G.

Proof: Suppose $\mathrm{S}$ is not a minimal H-dominating set of G. Since $\mathrm{S}$ is an H-dominating set of G, it properly contains a minimal $\mathrm{H}$-dominating subset of $\mathrm{G}$ say $\mathrm{S}_{1}$. Since $\mathrm{S}_{1}$ is an H-dominating set of $\mathrm{G}$ not containing $\mathrm{v}, \mathrm{S}_{1}$ is also an $\mathrm{H}$-dominating set of $G \backslash v$. Thus $\mathrm{S}$ is a minimal $\mathrm{H}$-dominating set of $\mathrm{G}$ $\backslash\{\mathrm{v}\}$ which properly contains an H-dominating set of $G \backslash v$. This is a contradiction. Thus $\mathrm{S}$ must be a minimal $\mathrm{H}$-dominating set of $\mathrm{G}$.

From the above Propositions we have the following theorem.

Theorem 2.1.3: If $\mathrm{G}$ is a hypergraph and $v \in V(G)$ then $\Gamma_{h}(G \backslash v) \leq \Gamma_{h}(G)$.

Proof: Let $\mathrm{S}$ be a $\Gamma_{h}$-set of $G \backslash v$ then either $\mathrm{S}$ is a minimal H-dominating set of $\mathrm{G}$ or $S \cup\{v\}$ is a minimal H-dominating set of $\mathrm{G}$. Therefore $\Gamma_{h}(G \backslash v) \leq \Gamma_{h}(G)$.

Theorem 2.1.4: Let $\mathrm{G}$ be a hypergraph and $v \in V(G)$ then the following statements are equivalent.

(1) $\Gamma_{h}(G \backslash v)=\Gamma_{h}(G)$.

(2) There is a $\Gamma_{h}$-set of $G \backslash v$ which is also $\Gamma_{h}$-set of G.

(3) every $\Gamma_{h}$-set of $G \backslash v$ is a $\Gamma_{h}$-set of G.

Proof: (1) $\Rightarrow(2)$ Let $\mathrm{S}$ be a $\Gamma_{h}$-set of $G \backslash v$. If $\mathrm{S}$ is not an H-dominating set of $\mathrm{G}$ then $S \cup\{v\}$ is a minimal H-dominating set of G. This will imply that $\Gamma_{h}(G \backslash v)<\Gamma_{h}(G)$. Which is not true. Therefore $\mathrm{S}$ must be an $\mathrm{H}$-dominating set of $\mathrm{G}$ and a minimal H-dominating set of $\mathrm{G}$. Therefore it must be $\Gamma_{h}$-set of G. Therefore (1) $\Rightarrow(2)$ is follows.

$(2) \Rightarrow(1)$. Let $\mathrm{S}$ be a $\Gamma_{h}$-set of $G \backslash v$ which is also an $\Gamma_{h}$-set of $\mathrm{G}$ then $\Gamma_{h}(G \backslash v)=|S|=\Gamma_{h}(G)$.

(1) $\Rightarrow(3)$. Let $\mathrm{S}$ be any $\Gamma_{h}$-set of $G \backslash v$. If $\mathrm{S}$ is not an H-dominating set of $\mathrm{G}$ then $S \cup\{v\}$ is a minimal H-dominating set of G. Therefore $\Gamma_{h}(G \backslash v)<|S \cup\{v\}|<\Gamma_{h}(G)$ this is a contradiction. Therefore $\mathrm{S}$ must be an H-dominating set of G. Thus $\mathrm{S}$ is a $\Gamma_{h}$-set of G.

(3) $\Rightarrow(1)$. If $\Gamma_{h}(G \backslash v)<\Gamma_{h}(G)$. There is a $\Gamma_{h}$-set of $G \backslash v$ which is not a $\Gamma_{h}$-set of $\mathrm{G}$ which contradicts (3). Therefore $\Gamma_{h}(G \backslash v)=\Gamma_{h}(G)$.

Example 2.1.5: Consider the hypergraph $G$ whose vertex set $V(G)=\{1,2,3,4,5,6,7,8,9,10,11\}$ and edges are $e_{1}=\{1,2,3,4\}, e_{2}=\{8,9,10,11\}, e_{3}=\{2,5,8\}, e_{4}=\{3,6,9\}, e_{5}=\{4,7,10\}$

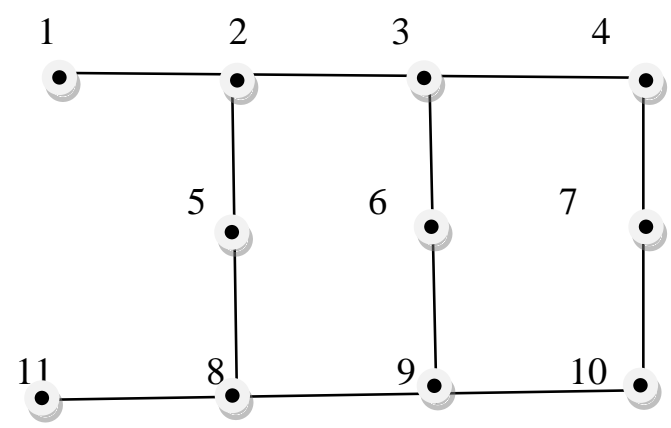

Consider the set $S=\{1,5,6,7,8,9,10\}$ it is obvious that $S$ is a minimal dominating set with maximum cardinality. This means that $\mathrm{S}$ is a $\Gamma_{h}$-set of $\mathrm{G}$. Thus the upper H-domination number of $\mathrm{G}$ is 7 . 
Now remove vertex $v=11$ from this hypergraph to get the sub-hypergraph $G \backslash 11$ whose vertices are $\{1,2,3,4,5,6,7,8,9,10\}$ and edges are $e_{1}{ }^{\prime}=\{1,2,3,4\}, e_{2}^{\prime}=\{2,5,8\}, e_{3}{ }^{\prime}=\{3,6,9\}, e_{4}{ }^{\prime}=$ $\{4,7,10\}, e_{5}{ }^{\prime}=\{8,9,10\}$. In this hypergraph the above set $S$ is again $\Gamma_{h}$-set of $G \backslash 11$. And therefore the upper H-domination number of $G \backslash 11$ is 7 . Thus $\Gamma_{h}(G \backslash 11)=\Gamma_{h}(G)$.

Theorem 2.1.6: Let $\mathrm{G}$ be a hypergraph and $v \in V(G)$ suppose $\Gamma_{h}(G \backslash v)<\Gamma_{h}(G)$ then $\Gamma_{h}(G \backslash v)=\Gamma_{h}(G)-1$ if there is a $\Gamma_{h}$-set S of G such that $v \in S$.

Proof: Suppose there is $\Gamma_{h}$-set T of G containing $v$. First we prove that $T \backslash\{v\}$ is an H-dominating set of $G \backslash v$. Let $x$ be any vertex of $G \backslash v$ which is not in $T \backslash\{v\}$ then $\mathrm{x} \notin \mathrm{T}$. There is an edge $e$ of $\mathrm{G}$ such that $x \in e$ and $e \backslash\{x\}$ is a subset of $e$. Let $e^{\prime}=e \backslash\{v\}$. obviously $e^{\prime} \backslash\{x\}$ is a subset of $T \backslash\{v\}$. Thus $T \backslash\{v\}$ is an H-dominating set of $G \backslash v$.

Now we prove that $T \backslash\{v\}$ is a minimal H-dominating set of $G \backslash v$. Let $x$ be any vertex of $T \backslash\{v\}$. If there is no edge $e$ of G containing $x$ such that $e \subset \mathrm{T}$ then there is no edge $e$ 'of $G \backslash v$ containing $x$ such that $e^{\prime} \subset T \backslash\{v\}$. Therefore $x$ belongs to private $\mathrm{H}$-neighborhood of $x$ with respect to $\mathrm{T}$ in $\mathrm{G}$ then $x$ belongs to private H- neighborhood of $x$ with respect to $T \backslash\{v\}$ in $G \backslash v$. Suppose $x$ does not belongs to private $\mathrm{H}$-neighborhood of $x$ with respect to $\mathrm{T}$ in $\mathrm{G}$ therefore there is a vertex $w$ in $V(G) \backslash T$ such that $w$ belongs to private $\mathrm{H}$-neighborhood of $x$ with respect to $\mathrm{T}$ in $\mathrm{G}$ then $w$ is a vertex of $G \backslash v$ which is not in $T \backslash\{v\}$. There is an edge $e$ of G containing $w$ and $x$ such that $e \backslash\{x, w\}$ is a subset of T.

Let $h^{\prime}$ be any other edge of $G \backslash v$ containing $w$. Let $h$ be the edge of G such that $h \backslash\{v\}=h^{\prime}$, Now $h \backslash\{w\}$ contains a vertex which is not in T. Therefore $h^{\prime} \backslash\{w\}$ contains a vertex which is not in $T \backslash\{v\}$. Therefore $w$ is a private H-neighborhood of $x$ with respect to $T \backslash\{v\}$ in the sub-hypergraph $G \backslash v$. Thus $T \backslash\{v\}$ is a minimal H-dominating set of $G \backslash v$.

Since $\Gamma_{h}(G \backslash\{v\})<\Gamma_{h}(G), T \backslash v$ must be a $\Gamma_{h}$-set of $G \backslash v$. Therefore

$\Gamma_{h}(G \backslash v)=|T \backslash\{v\}|=|T|-1=\Gamma_{h}(G)-1$.

\subsection{Partial sub-hypergraph}

Proposition 2.2.1: Let $\mathrm{G}$ be a hypergraph and $v \in V(G)$. Let $\mathrm{S}$ be a subset of $\mathrm{V}(\mathrm{G})$ not containing $v$

(1) If $\mathrm{S}$ is an H-dominating set of $\mathrm{G}$ then $\mathrm{S}$ is an H-dominating set of $G \backslash v$.

(2) If $\mathrm{S}$ is an H-dominating set of $G \backslash v$ then either $\mathrm{S}$ is an H-dominating set of $\mathrm{G}$ or $S \cup\{v\}$ is an $\mathrm{H}$-dominating set of $\mathrm{G}$.

Proof: (1) Suppose $\mathrm{S}$ is an H-dominating set of G. Let $x$ be a vertex of $G \backslash v$ which is not in $\mathrm{S}$. There is an edge $e$ of $\mathrm{G}$ containing $x$ such that $e \backslash\{x\}$ is a subset of $\mathrm{S}$ then $e$ does not contain $\mathrm{v}$ and therefore $e$ is an also edge of $G \backslash v$. Thus $\mathrm{S}$ is an H-dominating set of $G \backslash v$.

(2) Suppose $\mathrm{S}$ is an H-dominating set of $G \backslash v$. If $\mathrm{S}$ is an H-dominating set of $\mathrm{G}$ then the statement is true. Otherwise there is an edge $e$ containing $v$ such that $e \backslash\{v\}$ contains vertices which are not in $\mathrm{S}$. Consider the set $S_{1}=S \cup\{v\}$ then obviously $S_{1}$ is an H-dominating set of G.

Proposition 2.2.2: Let $\mathrm{G}$ be a hypergraph and $v \in V(G)$. Let $\mathrm{S}$ be a subset of $V(G)$ not containing $v$. If $\mathrm{S}$ is an $\mathrm{H}$-dominating set of $\mathrm{G}$ and it is also a minimal $\mathrm{H}$-dominating set of $G \backslash v$ then $\mathrm{S}$ is a minimal H-dominating set of $\mathrm{G}$.

Proof: From the previous theorem we already noted that $\mathrm{S}$ is an H-dominating set of $G \backslash v$. Let $x \in S$. Suppose there is no edge of $G \backslash v$ containing $x$ and subset of $\mathrm{S}$. (because $\mathrm{v} \notin \mathrm{S}$ ) Thus if $x$ belongs to private H-neighborhood of $x$ with respect to $\mathrm{S}$ in $G \backslash v$ then $x$ belongs to private H-neighborhood of $x$ with respect to $\mathrm{S}$ in $\mathrm{G}$.

Let $w$ be a vertex of $G \backslash v$ such that $w \neq x$ and $w$ belongs to private H-neighborhood of $x$ with respect to $\mathrm{S}$ in $G \backslash v$. Therefore there is an edge $h$ of $G \backslash v$ such that $x, w \in h$ and $h \backslash\{x, w\}$ is a subset 
of $\mathrm{S}$. The edge $h$ is also an edge of $\mathrm{G}$. Thus there is an edge $h$ of $\mathrm{G}$ containing $x$ and $w$ such that $h \backslash\{x, w\}$ is a subset of $\mathrm{S}$.

Let $f$ be an edge of G containing $w$ such that $f \neq h$. If $v \in f$ then $f$ contains a vertex of G which is not in S. If $v$ does not belong to $f$ then $f$ is an edge of $G \backslash v$ and since $w$ is a private H-neighbor of $x$ in $G \backslash v, f$ contains a vertex of $G \backslash v$ which is not in $\mathrm{S}$.

Thus it is proved that w belongs to private H-neighborhood of $x$ with respect to $\mathrm{S}$ in $\mathrm{G}$ also thus it is clear that for every $x$ in S private neighborhood of $x$ with respect to $x$ in $\mathrm{G}$ is a nonempty set. Thus $\mathrm{S}$ is a minimal $\mathrm{H}$-dominating set of $\mathrm{G}$.

Proposition 2.2.3: Let $\mathrm{G}$ be a hypergraph and $v \in V(G)$ then $\Gamma_{h}(G \backslash v) \leq \Gamma_{h}(G)$.

Proof: Let $S_{1}$ be a $\Gamma_{h}-$ set of $G \backslash v$. If $S_{1}$ is an H-dominating set of $\mathrm{G}$ then $S_{1}$ is a minimal Hdominating set of G. Therefore $\Gamma_{h}(G \backslash v)=\left|S_{1}\right| \leq \Gamma_{h}(G)$. If $S_{1}$ is not an H-dominating set of $\mathrm{G}$ then $S=S_{1} \cup\{v\}$ is a minimal H-dominating set of G and therefore $\Gamma_{h}(G \backslash v)=\left|S_{1}\right| \varangle S_{1} \cup\{v\} \mid \leq \Gamma_{h}(G)$. Thus from both the above cases $\Gamma_{h}(G \backslash v) \leq \Gamma_{h}(G)$.

Theorem 2.2.4: Let $\mathrm{G}$ be a hypergraph and $v \in V(G)$ then $\Gamma_{h}(G \backslash v)=\Gamma_{h}(\mathrm{G})$ if and only if there is a $\Gamma_{h}$-set $\mathrm{S}$ of $\mathrm{G}$ such that $v \notin S$.

Proof: Suppose $\Gamma_{h}(G \backslash v)=\Gamma_{h}(\mathrm{G})$. Let $S_{1}$ be a $\Gamma_{h}(\mathrm{G} \backslash \mathrm{v})$ If $S_{1}$ is not an H-dominating set of $\mathrm{G}$ then $S_{1} \cup\{v\}$ is a minimal H-dominating set of G. This will implies that $\Gamma_{h}(G \backslash v)=\left|S_{1}\right|<\left|S_{1} \cup\{v\}\right|$ $\leq \Gamma_{h}(G)$. This is a contradiction and therefore $S_{1}$ must be a minimal H-dominating set of G. Since $\Gamma_{h}(G \backslash v)=\Gamma_{h}(\mathrm{G}), S_{1}$ must be a $\Gamma_{h}$-set of $\mathrm{G}$ and it does not contain the vertex v.

Conversely suppose there is a $\Gamma_{h}$-set $\mathrm{S}$ of $\mathrm{G}$ which does not contains the vertex v. Then $\mathrm{S}$ is a minimal H-dominating set of $G \backslash v$. Therefore $\Gamma_{h}(\mathrm{G})=|\mathrm{S}| \leq \Gamma_{h}(G \backslash v)$. Since it is always true that $\Gamma_{h}(G \backslash v) \leq \Gamma_{h}(G)$, it follows that $\Gamma_{h}(G \backslash v)=\Gamma_{h}(\mathrm{G})$.

Corollary 2.2.5: Let $\mathrm{G}$ be a hypergraph and $v \in V(G)$ then $\Gamma_{h}(G \backslash v)<\Gamma_{h}(G)$ if and only if $v \in S$, for every $\Gamma_{h}-$ set $\mathrm{S}$ of $\mathrm{G}$.

Example 2.2.6: Consider the hypergraph $G$ whose vertex set $V(G)=\{0,1,2,3,4\}$ and edges are $e_{1}=\{1,0,2\}, e_{2}=\{3,0,4\}$

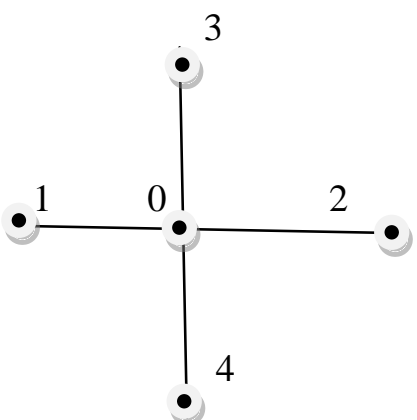

Obviously the set $S=\{1,2,3,4\}$ is a $\Gamma_{h}$-set of $\mathrm{G}$ and therefore the upper H- domination number of $G$ is 4 .

Now consider the sub-hypergraph $G \backslash 0$. The vertex set of this hypergraph is $\{1,2,3,4\}$ and edges are $e_{1}{ }^{\prime}=\{1,2\}, e_{2}{ }^{\prime}=\{3,4\}$. The set $T=\{1,3\}$ is an upper H-dominating set of $G \backslash 0$. Therefore the upper H-domination number of $G \backslash 0$ is 2 . Therefore $\Gamma_{h}(G \backslash 0)<\Gamma_{h}(G)$.

Consider the partial sub-hypergraph $G \backslash 0$ whose vertex set is $\{1,2,3,4\}$ and edge set is empty. Obviously the set $S=\{1,2,3,4\}$ is an upper H-dominating set of $G \backslash 0$. And therefore $\Gamma_{h}(G \backslash 0)=4=\Gamma_{h}(G)$. 
Example 2.2.7: Consider the hypergraph $G$ whose vertices are $\{1,2,3,4,5\}$ and edges are $e_{1}=\{1,2,3\}, e_{2}=\{2,4,5\}$. 5

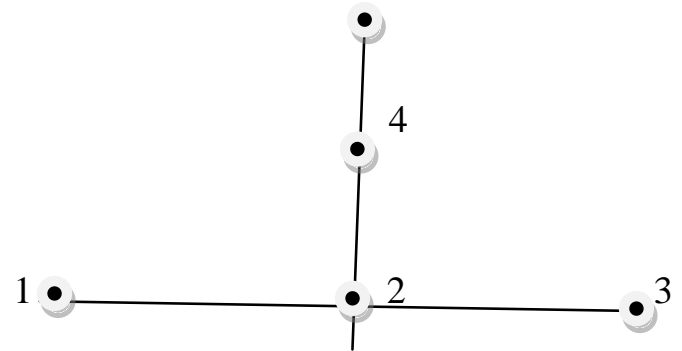

The set $S=\{1,3,4,5\}$ is a $\Gamma_{h}$-set of G. Therefore its upper H-domination number is 4 . Now consider the partial sub-hypergraph $G \backslash 5$. The vertices of this hypergraph are $\{1,2,3,4\}$ and it has only one edge namely $h=\{1,2,3\}$. Also this hypergraph has an isolated vertex namely 4 . The set $T=\{1,3,4\}$ is an upper $\mathrm{H}$-dominating set of $G \backslash 5$. Therefore its upper $\mathrm{H}$-domination number is 3 . Thus in this case $\Gamma_{h}(G \backslash 5)<\Gamma_{h}(G)$.

\section{Concluding Remarks}

We have considered the effect of removing a vertex on upper H-domination number of the hypergraph. It may be interesting to study the effect of removing an edge from the hypergraph. Further it may be possible to define edge addition in hypergraph and it may be possible to prove the corresponding results for edge addition.

\section{REFERENCES}

[1] B.D. Acharya, Domination in Hypergraphs, AKCE J. Graphs.Combin., No.2 (2007), pp.117-126

[2] C.BERGE, Hypergraphs, North-Holland Mathematical Library, New York Volume-45 (1989).

[3] D.K.Thakkar and K.N.Kalariya, H-domination in hypergraphs, International j. of Math.Sci. \& Engg. Appls. (IJMSEA), Vol.9. No.IV (December-2015). pp. 105-114. ISSN: 0973-9424.

[4] T.W.Haynes, S.T.Hedetniemi, P.J Slater, Domination in graphs Advanced Topics, Marcel Dekker, New York, 1998.

\section{AUTHOR's BIOGRAPHY}

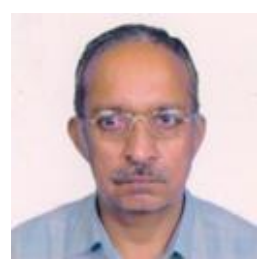

Dr.D.K.Thakkar, is in the department of the mathematics of Saurashtra University Rajkot his areas of interest are Graph theory, Topology and Discrete Mathematics. He has published over 45 research papers in various journals.

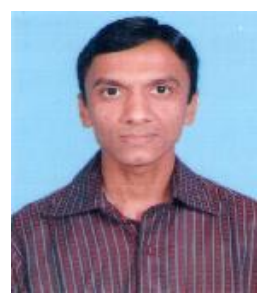

Mr.K.N.Kalariya, is in the mechanical department in Maths subject in V.V.P. Engg. College working as an assistant professor. My area of research work on the topic of hypergraphs. 